\title{
Non-resonant vibrational excitation of HOD and selective bond breaking
}

\author{
Dey, Diptesh; Henriksen, Niels Engholm
}

Published in:

Journal of Chemical Physics

Link to article, DOI:

$10.1063 / 1.5029548$

Publication date:

2018

Document Version

Peer reviewed version

Link back to DTU Orbit

Citation (APA):

Dey, D., \& Henriksen, N. E. (2018). Non-resonant vibrational excitation of HOD and selective bond breaking. Journal of Chemical Physics, 148(23), [234307]. https://doi.org/10.1063/1.5029548

\section{General rights}

Copyright and moral rights for the publications made accessible in the public portal are retained by the authors and/or other copyright owners and it is a condition of accessing publications that users recognise and abide by the legal requirements associated with these rights.

- Users may download and print one copy of any publication from the public portal for the purpose of private study or research.

- You may not further distribute the material or use it for any profit-making activity or commercial gain

- You may freely distribute the URL identifying the publication in the public portal

If you believe that this document breaches copyright please contact us providing details, and we will remove access to the work immediately and investigate your claim 
Diptesh Dey and Niels E. Henriksen ${ }^{1, \text { a) }}$

Department of Chemistry, Technical University of Denmark, Building 207, DK-2800 Kongens Lyngby, Denmark

(Dated: 4 June 2018)

This paper reports a time-dependent quantum mechanical wave packet study for bond-selective excitation and dissociation of HOD into the $\mathrm{H}+\mathrm{OD}$ and $\mathrm{D}+\mathrm{OH}$ channels in the first absorption band. Prior to excitation, the HOD molecule is randomly oriented with respect to a linearly polarized laser field and accurate static dipole moment and polarizability surfaces are included in the interaction potential. Vibrational excitation is obtained with intense, non-resonant $800 \mathrm{~nm}$ few-cycle excitation using dynamic Stark effect/impulsive Raman scattering. Dissociation is accomplished by another ultrashort VUV-laser excitation. A laser control scheme is designed with a train of simple, non-resonant laser pulses in order to enhance the selectivity between the fragmentation channels. The effect of carrier-envelope-phase of the ultrashort laser pulses is also investigated.

${ }^{a)}$ Electronic mail: neh@kemi.dtu.dk 


\section{Publishing INTRODUCTION}

The unique properties of laser light, like high intensity and the phase coherence of its frequency components are key to its application in femtosecond chemistry, ${ }^{1}$ i.e., the real-time observation and control of chemical reactions as they proceed from reactants to products. To that end, lasers can be applied to guide a system from a given initial state into a desired final state. ${ }^{2-5}$

The photodissociation dynamics of water and its deuterated derivative HOD,

$$
\mathrm{HOD} \longrightarrow\left\{\begin{array}{l}
\mathrm{H}+\mathrm{OD} \\
\mathrm{D}+\mathrm{OH}
\end{array}\right.
$$

in the first electronically excited state has attracted attention in the past two-three decades, both experimentally and theoretically. ${ }^{6-18}$ It has unique characteristics like the dissociation dynamics occurs on a purely repulsive excited state potential which is well separated from other excited states, the H-O-D bending mode is weakly coupled with the stretching modes, that is, the dynamics can be simulated within a two-dimensional model, etc. Thereby, it became an interesting molecule for studying unimolecular photodissociation and it can be considered as the "hydrogen atom" of laser-controlled bond-selective fragmentation. The dissociation is completed within about $20 \mathrm{fs}$, as recently detected in real time by employing 10-15 fs VUV pulses in a pump-probe setup. ${ }^{18,19}$

For HOD in its yibrational ground state, the branching ratio $(\mathrm{H}+\mathrm{OD}) /(\mathrm{D}+\mathrm{OH})$ has been studied as a function of the VUV-excitation frequency in the weak-field (one-photon) limit. ${ }^{7}$ The branching ratio is found to be $\gtrsim 2$ over the entire range of the first absorption band. Thus, a key target of the laser-controlled chemistry of the reaction in Eq. (1) is to achieve branching ratios less than $\sim 2$. Laser-controlled selective bond breaking in HOD can be achieved in, at least, three ways: (1) with two cw (continuous wave) lasers, where the first infrared laser vibrationally excites the molecule to one of its stationary (local mode) eigenstates, and the second resonant ultraviolet laser transfers the molecule to the first electronically excited state where it dissociates. This approach has been demonstrated in both experimental and theoretical studies; ${ }^{7-11}$ (2) using a similar but more general approach based on pulsed lasers, where the first laser pulse creates non-stationary vibrational states, followed by a properly timed resonant excitation to the dissociative excited electronic state; ${ }^{12-14}(3)$ with the application of an optimized intense VUV pulse directly to the 
Publishimglecule in its vibrational ground state. ${ }^{16}$

In the present work, we will consider the second of the above approaches, focusing on the creation of non-stationary vibrational states in HOD, which in previous theoretical studies were obtained via short-pulse resonant excitations. In these theoretical studies ${ }^{12-14}$ (see also Ref.[20]) it was assumed that the HOD molecule was pre-oriented prior to vibrational excitation. Although it is possible to orient a molecule, a scheme-without this requirement is obviously easier to implement experimentally. Thus, in this work we will consider HOD molecules which are randomly oriented, e.g. corresponding to the rotational ground state. Moreover, with recent advances in laser technology, few-cycle, ultrashort laser pulses have become available to the experimentalists. ${ }^{21}$ To that end, strong-field experiments were recently conducted on HOD with 2-cycle laser pulses of $800 \mathrm{~nm}$ wavelength ${ }^{22}$ (note that, due to very high intensities, cationic states of HOD were involved in the dissociation dynamics). The temporal duration of such a laser pulse is of the order of a few femtoseconds, i.e., shorter than any vibrational period in a molecule. These laser pulses are an optimal tool for the non-resonant dynamic Stark effect, equivalent to impulsive stimulated Raman scattering, for vibrational excitation. ${ }^{2-30}$ This motivates us to consider new possibilities of non-stationary vibrational excitation and selective bond breaking for the reaction in Eq. (1). The application of non-resonant laser pulses on a randomly oriented sample lends itself to a convenient experimental implementation.

To formulate this scenario, in short, we would like to understand the vibrational dynamics induced by these few-cycle, non-resonant laser pulses in comparison with the previous resonant vibrational excitation. This work will subsequently address the following questions: How will the vibrational excitation depend on the orientation of the molecule with respect to the polarization of the laser pulse? Can (bond-selective) vibrational excitation be obtained, in a simple manner? Does carrier-envelope-phase (CEP) of these ultrashort laser pulses play any role for the dynamics, as earlier reported in some other context? To what extent can we control the dissociation dynamics of HOD via the off-resonant vibrational pre-excitation?

In order to investigate these above mentioned questions, a time-dependent quantum mechanical wave packet calculation is performed by considering the two degrees of freedom along the H-O-D bond stretchings with the bond angle restricted to its equilibrium value. The initial nuclear wave packet (WP) of the electronic ground state has been chosen in a systematic manner to deal with the possibility of a random orientation of the molecule 
Publishiwgh respect to a linearly polarized laser field. Since, laser-molecule interaction with intense nonresonant pulses will be studied, the polarizability tensor components must to be taken into account in the interaction potential, along with an accurate dipole moment surface of water; both of which should deal with the orientation of the molecule. Following the laser induced vibrational dynamics in the ground state, an ultrafast-pulse excitation to the first excited state is carried out. Dissociation dynamics is analysed by-measuring the flux along the two fragmentation channels, from which the branching ratio can be determined.

An outline of this paper is as follows: In Sec. II, we briefly describe our theoretical approach which systematically incorporates the random orientation of the molecule with respect to a linearly polarized laser pulse. Results are presented and analysed in Sec. III. A summary of our major findings is given in Sec. IV.

\section{METHODOLOGY}

The photodissociation dynamics of HOD under the influence of 2-cycle, non-resonant laser pulses is studied by numerically solving the time-dependent Schrödinger equation in internal coordinates. ${ }^{12,31}$ Since the stretching and bending modes are only weakly coupled, a two degree of freedom model is considered where $\mathrm{O}-\mathrm{H}$ and $\mathrm{O}-\mathrm{D}$ bond stretchings are allowed and the bending is neglected. The kinetic energy operator can be written as

$$
\hat{T}=\frac{\hat{p}_{\mathrm{OH}}^{2}}{2 \mu_{\mathrm{OH}}}+\frac{\hat{p}_{\mathrm{OD}}^{2}}{2 \mu_{\mathrm{OD}}}+\frac{\hat{p}_{\mathrm{OH}} \hat{p}_{\mathrm{OD}}}{m_{\mathrm{O}}} \cos \theta_{\mathrm{eq}}
$$

where $p_{\mathrm{OH}}$ and $p_{\mathrm{OD}}$ are the conjugate momenta associated with the $\mathrm{O}-\mathrm{H}$ and $\mathrm{O}-\mathrm{D}$ bond lengths, respectively, $\mu_{\mathrm{OH}}=m_{\mathrm{O}} m_{\mathrm{H}} /\left(m_{\mathrm{O}}+m_{\mathrm{H}}\right)$ and $\mu_{\mathrm{OD}}=m_{\mathrm{O}} m_{\mathrm{D}} /\left(m_{\mathrm{O}}+m_{\mathrm{D}}\right)$ are the $\mathrm{OH}$ and OD reduced/masses, and $\theta_{\text {eq }}$ is the bending angle fixed at the equilibrium value of $104.52^{\circ}$

Under field-free conditions, we consider an isotropic distribution of the molecule in space. To systematically implement this in our code, we followed the idea of a rotated wave function. ${ }^{32}$ The initial nuclear WP is represented as a superposition of WPs, each with different orientation in space, in order to describe a state of zero total angular momentum. Thus,

$$
\Psi\left(r_{\mathrm{OH}}, r_{\mathrm{OD}}, \varphi, \theta, \chi\right)=\int_{\Omega} \psi\left(r_{\mathrm{OH}}, r_{\mathrm{OD}}\right) \delta\left(\varphi-\varphi_{0}\right) \delta\left(\theta-\theta_{0}\right) \delta\left(\chi-\chi_{0}\right) d \Omega_{0}
$$


Publishiwgere $\varphi, \theta$, and $\chi$ are Euler angles (to be specified below), $d \Omega_{0}=d \varphi_{0} d \chi_{0} \sin \theta_{0} d \theta_{0}$, and rotational-vibrational coupling has been neglected. The time-evolution operator is linear and it depends on the orientation of the molecule $\left(\varphi_{0}, \theta_{0}, \chi_{0}\right)$ due to the interaction with the laser field. That is, the dynamics is determined by propagating each term in the superposition of Eq. (3), one at a time, followed by an integration over all the orientations. In practice, the integral is discretized by a Gauss-Legendre quadrature.

The initial nuclear WP corresponding to one of the orientations is obtained by the time relaxation method, ${ }^{33}$ by propagating an arbitrarily chosen Gaussian WP on the ground electronic state potential energy surface ${ }^{12}$ in imaginary time. The random orientation is broken in the presence of the laser pulses, corresponding to rotational excitation, but rotational motion (alignment) is slow on the femtosecond time scale-of the dissociation dynamics ${ }^{29}$ and the molecule will, essentially, retain its random orientation upon dissociation.

Since laser pulses with Gaussian envelopes closely resemble experimental pulse shapes, ${ }^{22}$ the temporal profile of the applied laser electric field is chosen as

$$
E(t)=E_{0} \exp \left(-4 \ln 2\left(t-t_{0}\right)^{2} / \tau^{2}\right) \cos \left[\omega\left(t-t_{0}\right)+\phi\right]
$$

where the peak amplitude $\left(E_{0}\right)$ and the angular frequency $(\omega)$ corresponds to a $10^{14} \mathrm{~W} / \mathrm{cm}^{2}$ intense laser pulse of $800 \mathrm{~nm}$ wavelength. $\phi$ defines the CEP of the laser pulse, i.e., the temporal offset between the maxima of the pulse envelope and the optical cycle and the full width at half maximum (FWHM) pulse duration $(\tau)$ of 3.5 fs is chosen to mimic a 2-cycle optical pulse. This pulse duration is in fact well chosen in the present context of vibrational excitation in HOD, since it can be shown that for a Gaussian pulse with a fixed maximum intensity, the maximum vibrational amplitude of a harmonic oscillator is obtained for a pulse duration which is equal to the systems oscillation period divided by $\sqrt{2} \pi .^{28}$ The chosen peak intensity is high; if the undesired process of ionization should turn out to play a major role, although the pulse is very short, the pulse can be "stretched" into a train of pulses, as described in Sec. III.

With intense, non-resonant laser excitation, the electrons of the molecule become significantly perturbed, i.e., polarization forces start to play an important role. Therefore, we need to go beyond the static electric dipole approximation and also consider the first-order polarizability term in the interaction potential, which is basically the field induced dipole moment. ${ }^{28}$ Avila performed coupled cluster calculations with optimally designed basis set 
Publishiargd reported an ab initio, static, dipole polarizability $(\alpha)$ surface of water which reproduces experimental Raman spectra. ${ }^{34}$ In the present study, we have used this polarizability surface. Taking into account the orientation dependency, the interaction potential due to polarizability is,

$$
\begin{aligned}
H_{\text {ind }}= & -\frac{1}{2} E^{2}(t)\left[\sin ^{2} \theta\left(\alpha_{X X} \cos ^{2} \chi+\alpha_{Y Y} \sin ^{2} \chi\right)\right. \\
& \left.+\alpha_{Z Z} \cos ^{2} \theta-\alpha_{X Z} \cos \chi \sin \theta \cos \theta\right]
\end{aligned}
$$

similar to earlier reports. ${ }^{35}$ The body(molecule)-fixed components $\left(\alpha_{I J}\right)$ of the polarizability tensor are transformed to the space-fixed components by using the Euler rotation matrix following the widely used y-convention. ${ }^{36}$ Here, the transformation is being carried out by performing three successive rotations: First, a rotation of $\varphi$ about the vertical $z$-axis, which is followed by a rotation of $\theta$ about the $y$-axis in the new $S^{\prime}$ frame (referred to as the line of nodes), and finally a rotation of $\chi$ is conducted about the transformed $z$-axis in $S^{\prime \prime}$ frame (or the figure axis). $\theta$ and $\chi$ are also referred to as the polar and azimuthal Euler rotation angles, respectively. The body-fixed axes coincides with the space-fixed axes when the polar and azimuthal angles are both fixed at zero

A highly accurate $a b$ initio dipole moment $(\mu)$ surface of water is also available, as reported by the group of Tennyson. 37 They performed all-electron, internally contracted multi-reference configuration interaction calculations and found good agreement when tested against high precision experimental spectroscopic data. This dipole moment surface has been used in our calculation. Considering the orientation dependency, the interaction potential due to the static dipole is given by,

$$
H_{\text {stat }}=-E(t)\left[\mu_{Y} \cos \theta-\mu_{X} \cos \chi \sin \theta\right] .
$$

We followed a similar procedure as in the case of polarizability to transform the body(molecule)fixed components $\left(\mu_{I}\right)$ of the static dipole to its space-fixed components.

Dynamic Stark control (DSC) forms the basis of the laser control scheme ${ }^{25,26,28-30}$ where a non-resonant intense laser pulse induces vibrational dynamics of HOD in its ground electronic state, which followed by a properly timed ultrashort VUV-excitation undergoes a Franck-Condon (FC) transition to the totally repulsive first electronically excited state ${ }^{6}$ where it finally dissociates. The time evolution operator governs the propagation of the nuclear WP as follows

$$
\Psi(t+\Delta t)=\exp (-i \hat{H} \Delta t / \hbar) \Psi(t)
$$


Publishiwgere the action of exponential containing the kinetic energy operator is evaluated using a two dimensional fast Fourier transform algorithm, ${ }^{38}$ in combination with the split-operator method. ${ }^{39}$ Integration over the different orientations of the molecule corresponding to each of the Euler rotation angles is numerically implemented using an $N$-point Gauss-Legendre quadrature subroutine. ${ }^{40}$

The time- and space-integrated outgoing flux along a particular fragmentation channel $(\mathrm{H}+\mathrm{OD})$ is measured through a dividing line kept at $r_{\mathrm{OH}}=r_{\mathrm{f}}$ in the asymptotic region as (see, e.g., Ref.[14])

$$
\begin{aligned}
J_{\mathrm{H}+\mathrm{OD}} & =\hbar \int_{0}^{T} d t \int_{r_{\mathrm{OD}_{\text {min }}}^{r_{\mathrm{OD}} \max }} d r_{\mathrm{OD}} \\
& \times\left\{\frac{1}{\mu_{\mathrm{OH}}} \operatorname{Im}\left[\psi^{*} \frac{\partial \psi}{\partial r_{\mathrm{OH}}}\right]_{r_{\mathrm{f}}}+\frac{\operatorname{eos} \theta_{\mathrm{eq}}}{m_{\mathrm{O}}} \operatorname{Im}\left[\psi^{*} \frac{\partial \psi}{\partial r_{\mathrm{OD}}}\right]_{r_{\mathrm{f}}}\right\}
\end{aligned}
$$

where $\psi \equiv \psi\left(r_{\mathrm{OH}}, r_{\mathrm{OD}}, t\right)$ and the subscript $r_{\mathrm{f}}$ refers to the fixed value of $r_{\mathrm{OH}}$. A similar expression holds for the other fragmentation channel.

The wave packet is multiplied by a damping function ${ }^{41}$ along each of the spatial directions to avoid unphysical reflection from the edges of the grid. Numerical parameters used in this calculation are listed in Table I.

TABLE I. Numerical parameters used in WP propagation.

\begin{tabular}{lcl}
\hline \hline Parameters & Values & Description \\
\hline$\left(r_{\text {min }}, r_{\text {max }}\right) / a_{0}$ & $(1.0,11.71)$ & Range of $r$ values \\
$N_{r} \times N_{r}$ & $256 \times 256$ & Number of grid points \\
$r_{\mathrm{f}} / a_{0}$ & 7.5 & Flux analysis surface \\
$r_{\mathrm{d}} / a_{0}$ & 9.02 & Starting point of WP damping \\
$\Delta t / \mathrm{fs}$ & 0.02419 & Time step used in propagation \\
\hline \hline
\end{tabular}

\section{RESULTS AND DISCUSSION}

\section{A. Vibrational excitation}

The influence of non-resonant excitation on the vibrational dynamics of the HOD molecule in a field-free randomly oriented sample is studied in a step-wise manner. As described, 
Publishimgr interaction potential is based on separate contributions from static dipole and induced dipole components. First, we consider how the static dipole term will govern the vibrational dynamics of this molecule in its ground electronic state for non-resonant excitation. Fig. 1 reports the expectation values of the $\mathrm{OH}$ and $\mathrm{OD}$ bond lengths as a function of time, with an interaction potential originating only from the static dipole term. The effect of considering a randomly oriented sample compared to a pre-oriented one is understood by choosing certain fixed orientations of the molecule. Subplots (a) and (c) correspond to the situation where the polar Euler angle $\theta$ is first rotated through $0^{\circ}$ and $180^{\circ}$, respectively, while the subsequent operation to rotate the azimuthal angle $\chi$ is not carried out and is therefore fixed at $0^{\circ}$. Whereas, in subplots (b) and (d) the polar angle is first rotated by $90^{\circ}$ and then the azimuthal angle is varied from $0^{\circ}$ to $180^{\circ}$.

It is clear from all these subplots, that the extent of stretching (or compression) obtained with one of these orientations is exactly the same as the extent of compression (or stretching) obtained with its complimentary orientation. The excitation is less off-resonant for the $\mathrm{OH}$ bond oscillation, this gives the wider range of oscillations of the $\mathrm{OH}$ bond as compared to OD bond. Overall, as expected, the vibrational amplitudes are much smaller than for resonant excitation, i.e., when the central frequency of the laser is at resonance with a vibrational transition. ${ }^{12}$ The dependence on orientation is similar to the results reported in previous work. ${ }^{13,42}$ For a randomly oriented sample, we need to average over all angles. Therefore, the overall effect of the interaction potential due to the static dipole will ultimately wash out when stretching and compression are of equal magnitude. This is the case in the harmonic limit of small vibrational amplitudes. ${ }^{42}$

Following this, our next aim is to investigate how the vibrational dynamics will be governed by the polarizability component of the interaction potential for a randomly oriented molecule. In Fig. 2, the expectation values of the bond lengths as a function of time are plotted, considering the contribution arising from the polarizability term alone. Similar to the previous case, here also the vibrational dynamics at a few chosen orientations are reported. Now for $\theta$ equal to $0^{\circ}$ or $180^{\circ}$, identical results are obtained as expected from the form of Eq. (5). Thus, unlike the situation for the static-dipole interaction, the vibrational amplitudes associated with oppositely oriented molecules will not cancel out. Subplots (a) and (c) correspond to a situation where the polar angle $\theta$ is at first rotated anti-clockwise by $45^{\circ}, 120^{\circ}$, and $150^{\circ}$ with the azimuthal angle $\chi$ kept fixed at $0^{\circ}$. Whereas, in subplots 


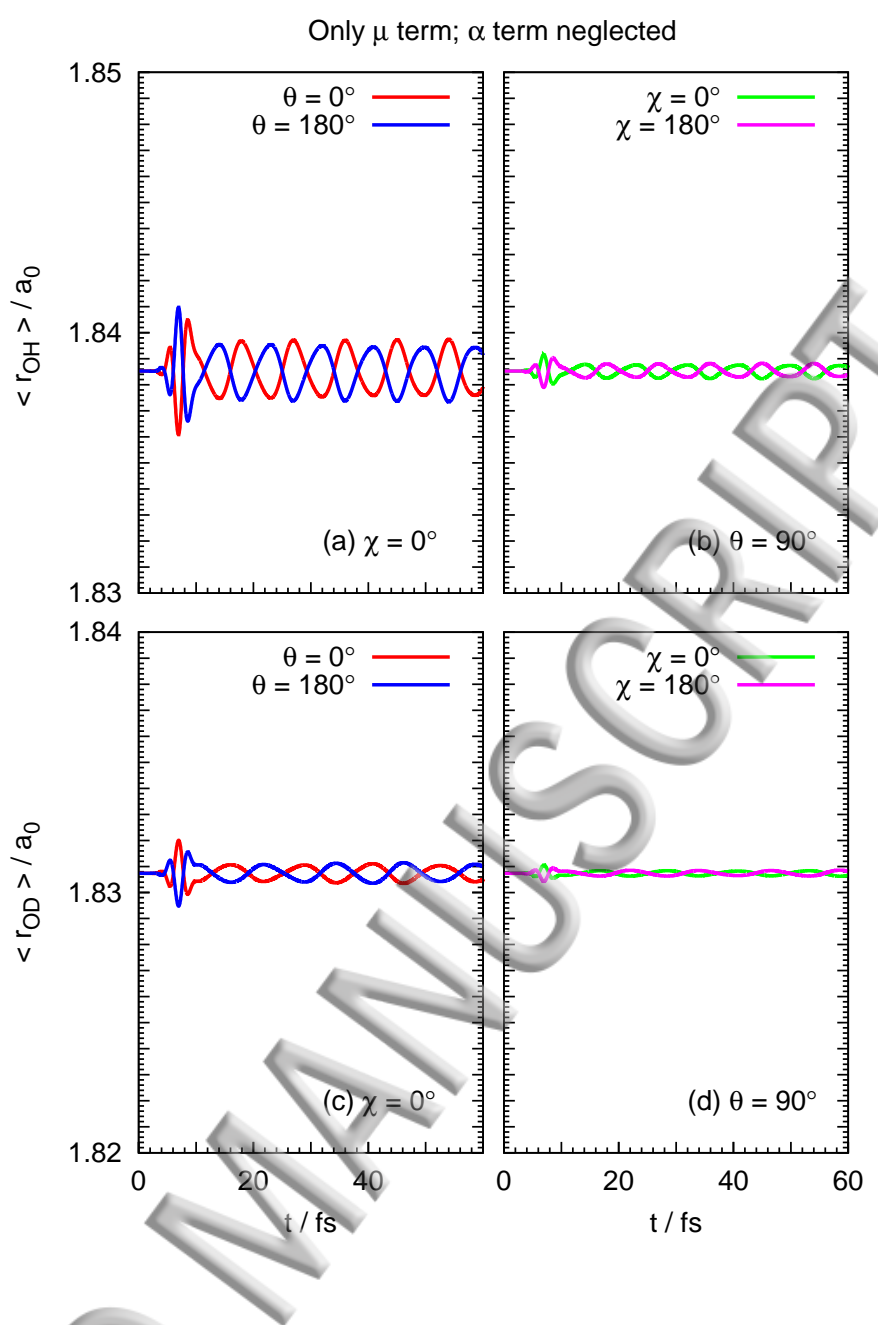

FIG. 1. Influence of the static electric dipole on the ground electronic state vibrational dynamics of HOD, at a few chosen orientations.

(b) and (d) the polar angle is first rotated anti-clockwise through $45^{\circ}$ with subsequent rotations of $0^{\circ}, 90^{\circ}$, and $180^{\circ}$ for the azimuthal angle. These results show that vibrational motion occurs of different extent depending on the particular angle chosen. Thereby, for an isotropic distribution of the sample, these stetchings (or compressions) will not cancel out and we will always be able to observe an average over all angles (see also Ref.[29]). Thus, for non-resonant laser excitation, it is the polarizability component of the interaction potential which will govern the vibrational dynamics and the contribution of the static dipole will not be observed.

For ultrashort laser pulses, often CEP has been found to influence the reaction outcome with significant alteration in the dissociation channels. ${ }^{43}$ In fact, the branching ratio of bond 


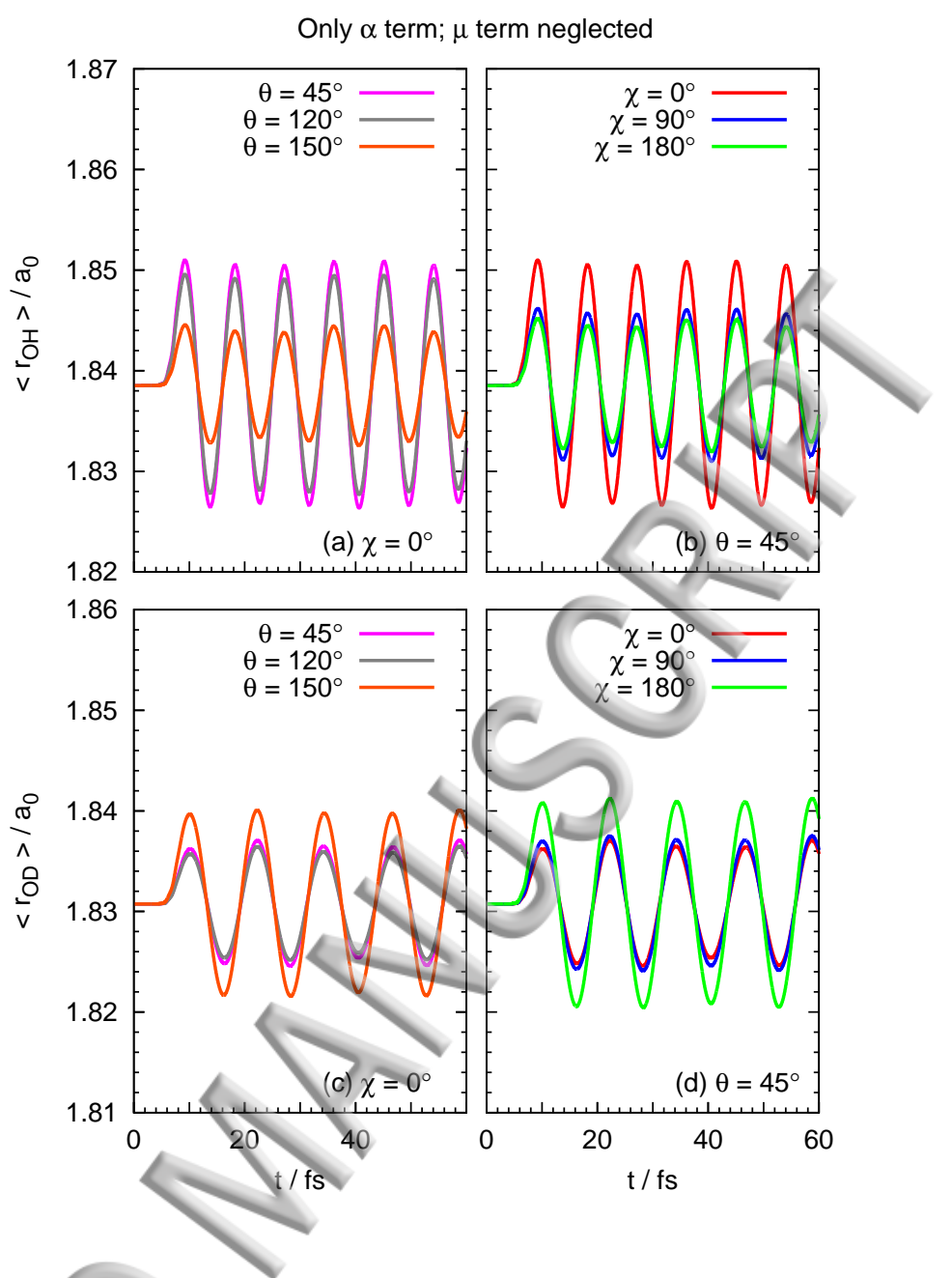

FIG. 2. Influence of the dipole polarizability on the ground electronic state vibrational dynamics of HOD, at a few chosen orientations.

breaking in HOD via molecular ionization was shown to be affected by laser CEP. ${ }^{22}$ Thereby, it is interesting to analyse whether the CEP can be used as a tool to govern the vibrational dynamics and eventually to control the photodissociation of this molecule. Fig. 3 presents the variation of average bond lengths as a function of time for different values of CEP, with contribution of the static dipole (subplots a and c) and induced dipole (subplots b and d) terms taken separately. The orientation of the molecule corresponding to the Euler polar and azimuthal angles are both fixed at zero, i.e., the body-fixed axes coincides with the space-fixed axes in this case. As revealed from these subplots (a)-(d), there is definitely a CEP dependency in the vibrational dynamics which arises from the dipole term (similar to earlier reports ${ }^{20,42}$ ) with almost no CEP dependency from the polarizability term. Moreover, 
Publishing on -resonant laser excitation is performed, the variation of the average bond lengths due to the dipole is significantly less as compared to the variation arising due to polarizability. Since, from Fig. 1 we came to the conclusion that the contribution of the static dipole will eventually vanish upon considering an isotropic distribution of the molecule, the same will be the fate for the CEP dependency of the vibrational excitation originating from the dipole term. Therefore, although we noted a CEP dependency for thesenon-resonant laser pulses to originate from the dipole term, they will ultimately cancel out upon considering an isotropic distribution of HOD. It is also important to point out that the CEP dependency reported in the joint experimental and theoretical study on bond selective fragmentation of HOD via ionization was observed at a much higher laser intensity $\left(10^{15} \mathrm{~W} / \mathrm{cm}^{2}\right)$, and the effect was found to almost wash out upon reducing the laser intensity $\left(7 \times 10^{14} \mathrm{~W} / \mathrm{cm}^{2}\right)$.

With a proper understanding of these factors that will govern the vibrational dynamics, we now plot the expectation value of the $\mathrm{OH}$ and $\mathrm{OD}$ bond lengths with time in Fig. 4, incorporating both the static dipole and induced dipole terms and performing the rotational average. The nature of these curves clearly indicate, according to our expectation, that the dipole term is not contributing anymore and the curves now closely resemble an average over the polarizability contributions. The laser pulse is also plotted alongside (in arbitrary scale) to visualize the onset of the vibrational dynamics. Comparing these non-resonant vibrational excitations with previous theoretical studies with resonant excitation, clearly suggest that the range of oscillations of the bond lengths is quite small. ${ }^{12}$ The reason is mainly due to the fact that for resonant excitation, the static dipole plays the major role for the marked effect, whereas, for non-resonant excitation it is only via the induced dipole or polarizability component. For further comparison we note that the uncertainties in the bond lengths are of the order of $0.1 a_{0}$. Thus, the mean displacements are clearly smaller than the uncertainties. From this plot we can also extract the characteristic vibrational time periods of motion for the $\mathrm{OH}$ and $\mathrm{OD}$ bonds which is 9.0 fs for the former and 12.2 fs for the latter one.

To further influence the vibrational excitation, we applied the simple concept of pulse trains, i.e., a set of identical laser pulses each of which is exactly similar to the single laser pulse previously used but with a proper time delay between each of them. It has been proved analytically ${ }^{28}$ that applying a train of laser pulses with a proper time spacing, which corresponds to an integer multiple of the time period of bond oscillations, leads to an increase 


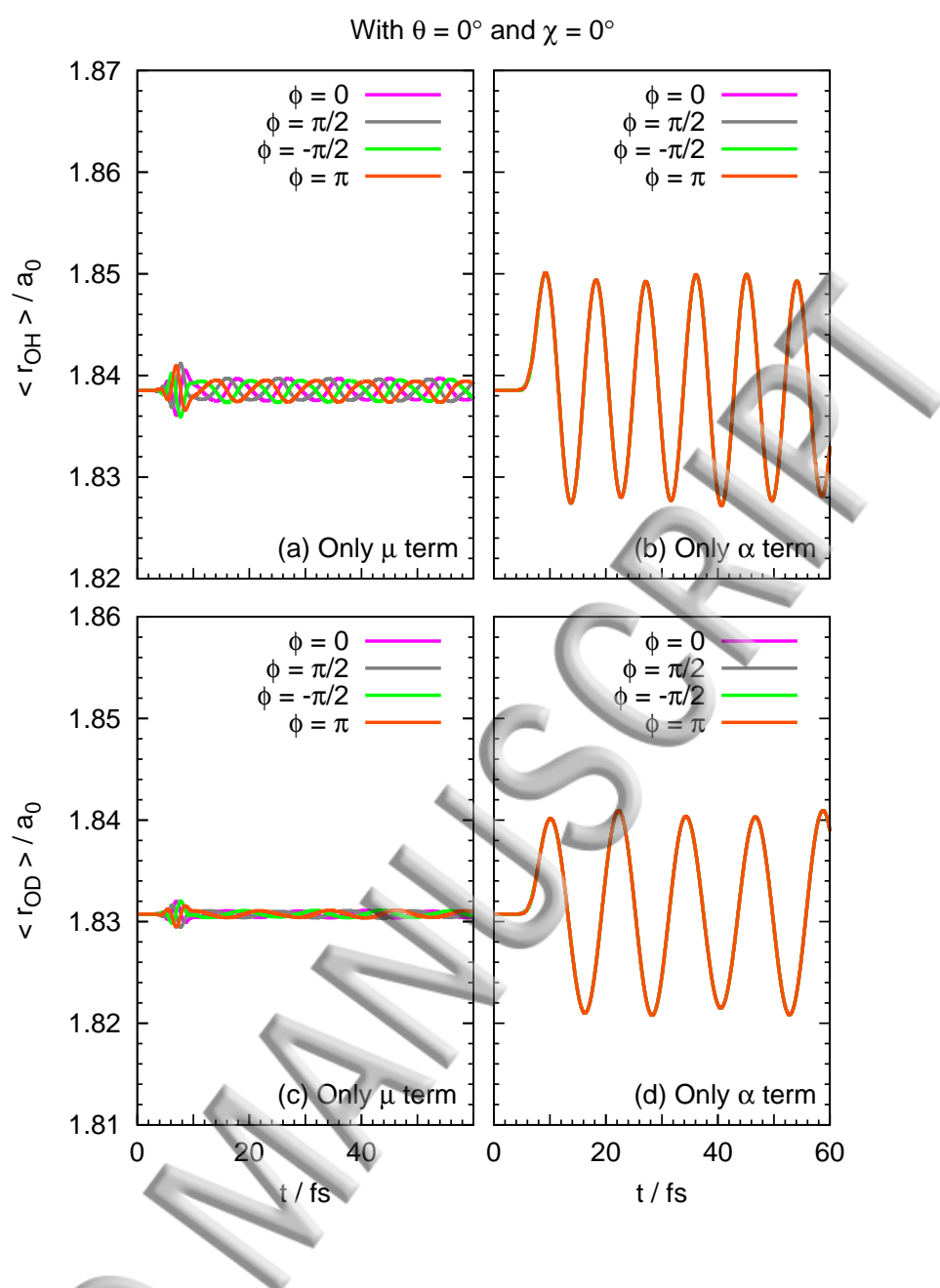

FIG. 3. CEP-dependency of the ultrashort laser pulses influencing the vibrational dynamics of HOD, as reflected by the variation of average bond lengths with time.

in the amplitude of the bond oscillations. Thus, the effect of each sub-pulse on the total amplitude will be additive within a harmonic model. Whereas, this may lead to no effective excitation of the system at all if the spacing is improper, say, with an integer multiple plus one half time period. This idea is applied in our present study and we first choose two sub-pulses. The separation between them is fixed at the time period of $\mathrm{OH}$ bond oscillations in one case, Fig. 5(a), and the time period of OD bond oscillations in other case, Fig. 5(b). From both the subplots (a) and (b), it is found that from the second pulse onwards there is a marked increase in the amplitude of the bond oscillations, where the increase of $\mathrm{OH}$ bond shows up in subplot (a) and the increase in the OD bond shows up in subplot (b) since the time spacings are chosen accordingly. Moreover, in subplot (b), we find that the $\mathrm{OH}$ bond 


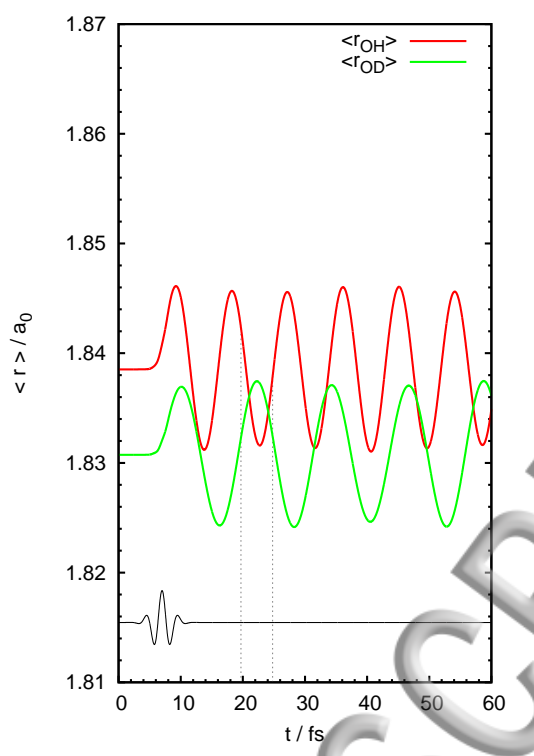

FIG. 4. Expectation value of the bond lengths as a function of time upon single pulse excitation. The laser pulse shown alongside is in arbitrary scale. The vertical dashed lines are at $19.7 \mathrm{fs}$ and $24.8 \mathrm{fs}$, see discussion in the text concerning photodissociation.

oscillations are reduced due to the improper time spacing, which might ultimately vanish if we choose an integral multiple ( $\gg 2$ ) plus one half period according to the analytical model.

\section{B. Photodissociation}

With the vibrationally excited molecule, some degree of selective bond breaking can be easily achieved upon application of a second laser pulse which will excite HOD to its first electronically/excited dissociative state. Fig. 4 already suggested that a single non-resonant laser pulse is unable to produce as much variation in the average bond lengths as can be obtained from resonant excitations, here we study therefore the extent of control that can be achieved. Exciting the oscillating HOD at different times with a properly timed $\delta$-pulse, we indeed found a variation in the flux along the two channels and in the branching ratio. A $\delta$-pulse is used for convenience but it should be noted that an ultrashort pulse with a finite pulse duration, of a few femtoseconds, will give similar results. ${ }^{12}$ With a $\delta$-pulse timed at 24.8 fs (see Fig. 4), for example, following the non-resonant excitation gives $76.7 \%$ of flux in the $\mathrm{H}+\mathrm{OD}$ channel and $22.8 \%$ in the other channel as revealed in Fig. 6(a), resulting in 

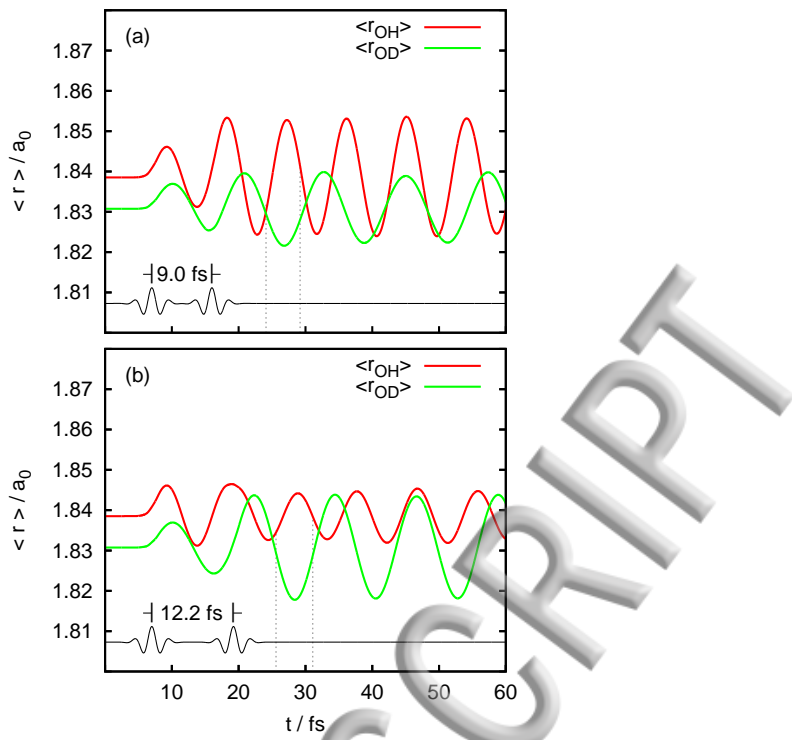

FIG. 5. Same as in Fig. 4, except that the laser pulse now consists of two similar sub-pulses with a separation of (a) 9.0 fs and (b) $12.2 \mathrm{fs}$, respectively. The vertical dashed lines are related to the discussion in the text concerning photodissociation.

a branching ratio of 3.36 which is basically the upper limit that can be reached. Whereas, if it is timed at $19.7 \mathrm{fs}$ (see Fig. 4) after the non-resonant excitation, $72.1 \%$ of flux in the $\mathrm{H}+\mathrm{OD}$ channel and $27.5 \%$ in the $\mathrm{D}+\mathrm{OH}$ channel are obtained as shown in Fig. 6(b) with a branching ratio of 2.62 as the lower limit. It is observed from Fig. 4 that at 19.7 fs, the oscillating OD bond is close to its equilibrium value, i.e., the average momentum is at its maximum leading to OD-bond stretching, whereas bond compression is observed for $\mathrm{OH}$. To that end, we note that the small displacements from the equilibrium position are well described by a harmonic oscillator and the average momentum is proportional to the time derivative of the average position. It has previously been noticed that the momentum distribution of the initial state plays an important role for the branching ratio in the photodissociation of HOD. ${ }^{12,13,15}$ To compare how the branching ratios are affected by the non-resonant vibrational excitation, we did a $\delta$-pulse excitation of the initial nuclear WP without shining the non-resonant laser pulse first and noted $74.5 \%$ of flux in the $\mathrm{H}+$ OD channel and $25.1 \%$ of flux in the $\mathrm{D}+\mathrm{OH}$ channel, with a branching ratio of 2.97. This brings us to the conclusion that there is indeed a small variation in the branching ratio of approximately $\pm 10 \%$. 


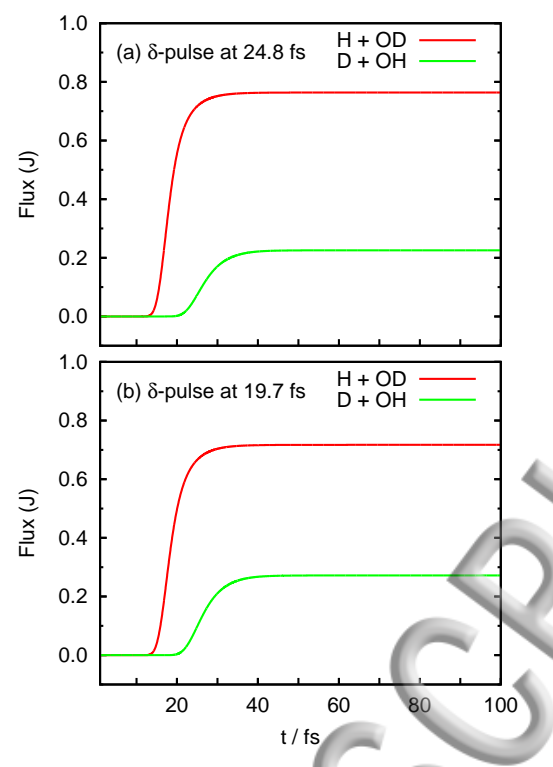

FIG. 6. Flux accumulated along the two dissociation channels as a function of time for $\delta$-pulse excitations at (a) $24.8 \mathrm{fs}$ and (b) $19.7 \mathrm{fs}$ with the vibrational excitation of Fig. 4. The time axes specify the time measured relative to the $\delta$-pulse.

But of course, the possibility of utilizing the larger vibrational amplitudes obtained with laser pulse trains should not be neglected. Therefore, the vibrational oscillations obtained in Fig. 5 are also tested for the degree of selective bond dissociation of HOD with $\delta$-pulse excitations at different instants of time. A branching ratio of 3.66 and 2.42 are obtained for $\delta$-pulse excitation timed at $24.1 \mathrm{fs}$ and $29.2 \mathrm{fs}$, respectively with a pulse separation of $9.0 \mathrm{fs}$ from Fig. 5(a); and branching ratio of 3.58 and 2.47 are observed for $\delta$-pulse excitations at $25.6 \mathrm{fs}$ and $31.1 \mathrm{fs}$, respectively with a pulse separation of $12.2 \mathrm{fs}$ as in Fig. 5(b). The effect on the branching ratio is now more pronounced than with just a single non-resonant laser excitation. Also from an experimental perspective, these simple laser pulse trains can be easy to achieve. The quite similar maximum/minimum branching ratios obtained from the two vibrating wave packets in Fig. 5 show again that the initial momentum distribution plays an important role in the subsequent fragmentation dynamics. Thus, in this case the momenta plays a larger role than the (small) displacement of atoms leading to bond stretches.

Application of laser pulse trains with two sub-pulses then brings us to the question of whether it might be possible to obtain a branching ratio less than 2 (see the discussion in Sec. I) and ultimately obtain selective breaking of the OD bond. To search for an answer, 

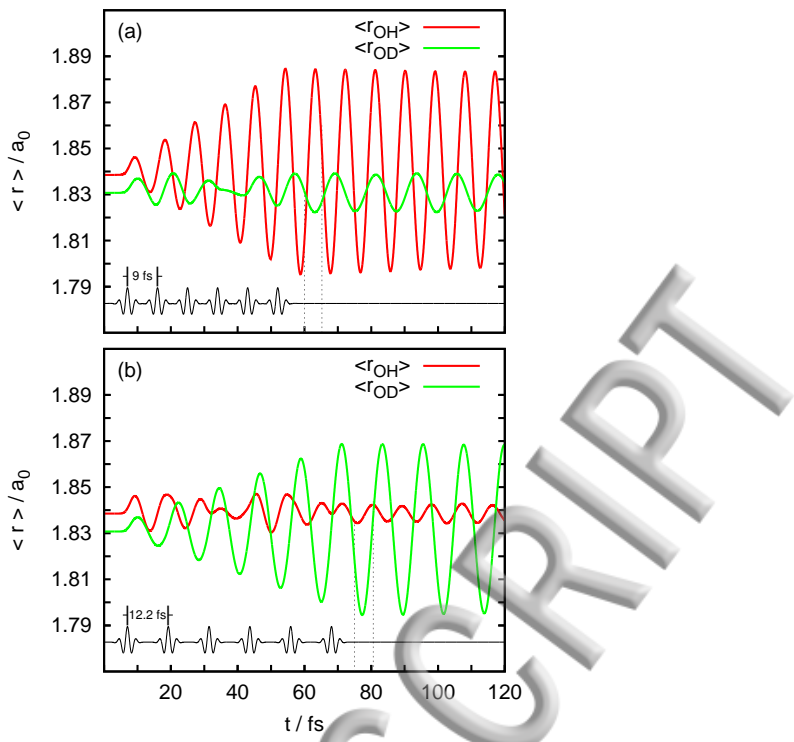

FIG. 7. Same as in Fig. 4, except that the laser pulse now consists of six similar sub-pulses with a separation of (a) 9.0 fs and (b) $12.2 \mathrm{fs}$, respectively. The vertical dashed lines are related to the discussion in the text concerning photodissociation.

we repeated our calculations upon addition of more sub-pulses in gradual steps and found that with a train of six sub-pulses, a branching ratio less than 2 can be obtained. As shown in Fig. 7, the picture is similar to the two-pulse case but with larger amplitudes. With a pulse separation of $9.0 \mathrm{fs}$, a branching ratio of 1.96 can be obtained with $\delta$-pulse excitation timed at $65.2 \mathrm{fs} ;$ a branching ratio of 4.7 is also noted for $\delta$-pulse excitation timed at $60.0 \mathrm{fs}$. With a pulse separation of $12.2 \mathrm{fs}$, a branching ratio of 1.96 can be obtained with $\delta$-pulse excitation timed at $80.6 \mathrm{fs}$; a branching ratio of 4.3 is obtained for $\delta$-pulse excitation timed at $75.0 \mathrm{fs}$. Again it is observed that the momenta plays a crucial role.

To summarize, the lower limit of branching ratios obtained with non-resonant laser excitation accompanied by a $\delta$-pulse are tabulated in Table II, along with the fluxes accumulated in the respective channels. It should be emphasized that these values only represent the lower limits which can be obtained with the investigated simple pulse trains. Thus, application of pulse trains with more than six sub-pulses or more advanced pulse shaping will lead to extended controllability. 
\$ 13 LE II. Lower limit of branching ratios obtained with non-resonant vibrational excitation followed by a $\delta$-pulse. The time delay between sub-pulses is $12.2 \mathrm{fs}$.

\begin{tabular}{cccc}
\hline \hline Non-resonant laser $(\delta$-pulse at) & $\mathrm{H}+\mathrm{OD}$ & $\mathrm{D}+\mathrm{OH}$ & $\frac{\mathrm{H}+\mathrm{OD}}{\mathrm{D}+\mathrm{OH}}$ \\
\hline$-(0 \mathrm{fs})$ & 0.745 & 0.251 & 2.97 \\
1 Pulse $(19.7 \mathrm{fs})$ & 0.721 & 0.275 & 2.62 \\
2 Pulses $(31.1 \mathrm{fs})$ & 0.708 & 0.286 & 2.47 \\
6 Pulses $(80.6 \mathrm{fs})$ & 0.649 & 0.331 & 1.96 \\
\hline
\end{tabular}

\section{SUMMARY AND CONCLUSION}

In this paper, we reported a time-dependent quantum dynamical WP study of nonresonant vibrational excitation of HOD followed by VUV photodissociation, with the aim of controlling the branching ratio between the channels $\mathrm{H}+\mathrm{OD}$ and $\mathrm{D}+\mathrm{OH}$. The current thrust for generation and use of ultrashort, few-cycle pulses by experimentalists motivated our study of the application of such pulses for vibrational excitation. Ultrashort non-resonant laser pulses can create non-stationary vibrational excitation, i.e., a vibrational wave packet via the dynamic Stark effect, which accompanied by a properly timed ultrashort VUV pulse transfers population from the ground electronic state to the repulsive first excited state where the molecule finally dissociates. The initial state represented an isotropic distribution of HOD in order to deal with a randomly oriented molecule in space and the laser-matter interaction included accurate static dipole and polarizability surfaces.

As a major finding, it turned out that the effect of the static dipole term ultimately washes out due to the rotational average whereas interaction via the induced dipole leads to vibrational excitations, although the amplitude indeed is smaller in comparison with similar resonant excitations. However, if we apply a train of ultrashort few-cycle pulses with proper time delay between the sub-pulses, then sufficient vibrational amplitude/momentum can be created, such that a desired bond selectivity can be obtained in the photodissociation of HOD. Furthermore, our studies showed that the CEP of the ultrashort laser pulses play no role in the vibrational excitation when a randomly oriented sample is considered.

Non-resonant excitation via dynamic Stark effect/impulsive Raman scattering is an attractive tool from an experimental point of view because, e.g., $800 \mathrm{~nm}$ femtosecond pulses are 
Publishiwglely available in contrast to resonant excitation sources. The utility of this non-resonant excitation mechanism has already been demonstrated in the case of rotational excitation. ${ }^{35}$ For vibrational excitation, as in the present work, it is clear that the effect of a single pulse is modest, pulse trains or more advanced pulse shaping are required in order to create substantial amplitudes. The utility of pulse shaping in this context has been demonstrated in recent works. ${ }^{28-30}$

\section{ACKNOWLEDGMENTS}

The research leading to these results has received funding from the European Union's Horizon 2020 research and innovation programme under the Marie Sklodowska-Curie grant agreement no. 713683 (COFUNDfellowsDTU).

\section{REFERENCES}

${ }^{1}$ A. H. Zewail, Angew. Chem. Int. Ed. 39, 2586 (2000).

${ }^{2}$ A. Assion, T. Baumert, M. Bergt, T. Brixner, B. Kiefer, V. Seyfried, M. Strehle, and G. Gerber, Science 282, 919 (1998).

${ }^{3}$ N. E. Henriksen, Chem. Soc. Rev. 31, 37 (2002).

${ }^{4}$ C. Brif, R. Chakrabarti, and H. Rabitz, New J. Phys. 12, 075008 (2010).

${ }^{5}$ M. Shapiro and P. Brumer, Quantum Control of Molecular Processes (Wiley, New York, 2012).

${ }^{6}$ V. Engel, R. Schinke, and V. Staemmler, J. Chem. Phys. 88, 129 (1988).

${ }^{7}$ V. Engel and R. Schinke, J. Chem. Phys. 88, 6831 (1988).

${ }^{8}$ D. G. Imre and J. Zhang, Chem. Phys. 139, 89 (1989).

${ }^{9}$ J.Zhang, D. G. Imre and J. H. Frederick, J. Phys. Chem. 93, 1840 (1989).

${ }^{10}$ R. L. Vander Wal, J. L. Scott, and F. F. Crim, J. Chem. Phys. 92, 803 (1990); I. Bar, Y.

Cohen, D. David, S. Rosenwaks, and J. J. Valentini, J. Chem. Phys. 93, 2146 (1990).

${ }^{11}$ M. Sharma, S. Adhikari, and M. K. Mishra, J. Chem. Phys. 127, 024305 (2007).

${ }^{12}$ B. Amstrup and N. E. Henriksen, J. Chem. Phys. 97, 8285 (1992).

${ }^{13}$ N. Elghobashi, P. Krause, J. Manz and M. Oppel, Phys. Chem. Chem. Phys. 5, 4806 (2003). 
Publishiłtg. K. Shandilya, S. Sen, T. Sahoo, S. Talukder, P. Chaudhury, and S. Adhikari, J. Chem. Phys. 139, 034310 (2013).

${ }^{15}$ N. E. Henriksen, K. B. Møller and V. Engel, J. Chem. Phys. 122, 204320 (2005).

${ }^{16}$ A. K. Tiwari, K. B. Møller and N. E. Henriksen, Phys. Rev. A 78, 065402 (2008).

${ }^{17}$ S. Talukder, P. Chaudhury, and S. Adhikari, Mol. Phys. 115, 1786 (2017).

${ }^{18}$ A. Baumann, S. Bazzi, D. Rompotis, O. Schepp, A. Azima, M. Weiland, D. PopovaGorelova, O. Vendrell, R. Santra and M. Drescher, Phys. Rev. A 96, 013428 (2017).

${ }^{19}$ S. A. Trushin, W. E. Schmid and W. Fuß, Chem. Phys, Lett. 468, 9 (2009).

${ }^{20}$ N. Elghobashi-Meinhardt, L. González, I. Barth, and T. Seideman, J. Chem. Phys. 130, 024310 (2009).

${ }^{21}$ T. Brabec and F. Krausz, Rev. Mod. Phys. 72, 545 (2000).

${ }^{22}$ D. Mathur, K. Dota, D. Dey, A. K. Tiwari, J. A. Dharmadhikari, and A.K. Dharmadhikari, J. Chem. Phys. 143, 244310 (2015).

${ }^{23}$ A. M. Weiner, D. E. Leaird, G. P. Wiederrecht, and K. A. Nelson, J. Opt. Soc. Am. B 8, 1264 (1991).

${ }^{24}$ R. A. Bartels, S. Backus, M. M. Murnane, and H. C. Kapteyn, Chem. Phys. Lett. 374, $326(2003)$.

${ }^{25}$ B. J. Sussman, D. Townsend, M. Y. Ivanov, and A. Stolow, Science 314, 278 (2006).

${ }^{26}$ B. J. Sussman, Am. J.Phys. 79, 477 (2011).

${ }^{27}$ L. Christensen, et al., Phys. Rev. Lett. 113, 073005 (2014).

${ }^{28}$ E. F. Thomas and N. E. Henriksen, J. Chem. Phys. 144, 244307 (2016).

${ }^{29}$ C. C. Shu, E. F. Thomas, and N. E. Henriksen, Chem. Phys. Lett. 683, 234 (2017).

${ }^{30}$ E. F. Thomas and N. E. Henriksen, J. Phys. Chem. Lett. 8, 2212 (2017).

${ }^{31}$ D. Dey and A. K. Tiwari, J. Phys. Chem. A 120, 2629 (2016).

${ }^{32}$ N. E. Henriksen and E. J. Heller, J. Chem. Phys. 91, 4700 (1989).

${ }^{33}$ R. Kosloff and H. Tal-Ezer, Chem. Phys. Lett. 127, 223 (1986).

${ }^{34}$ G. Avila, J. Chem. Phys. 122, 144310 (2005).

${ }^{35}$ H. Stapelfeldt and T. Seideman, Rev. Mod. Phys. 75, 543 (2003).

36 H. Goldstein, Classical Mechanics (Addison Wesley, New York, 2000).

${ }^{37}$ L. Lodi, J. Tennyson, and O. L. Polyansky, J. Chem. Phys. 135, 034113 (2011).

${ }^{38}$ D. Kosloff, R. Kosloff, J. Comput. Phys. 52, 35 (1983).

${ }^{39}$ M. D. Feit, J. A. Fleck, Jr., and A. Steiger, J. Comput. Phys. 47, 412 (1982). 
Publishifigy. H. Press, S. A. Teukolsky, W. T. Vetterling, and B. P. Flannery, Numerical Recipes in Fortran 77: The Art of Scientific Computing (Cambridge University Press, Cambridge, 1992).

${ }^{41}$ S. Mahapatra and N. Sathyamurthy, J. Chem. Soc., Faraday Trans. 93, 773 (1997).

${ }^{42}$ M. Machholm and N. E. Henriksen, J. Chem. Phys. 111, 3051 (1999).

${ }^{43}$ V. Roudnev, B. D. Esry and I. Ben-Itzhak, Phys. Rev. Lett. 93, 163601 (2004). 

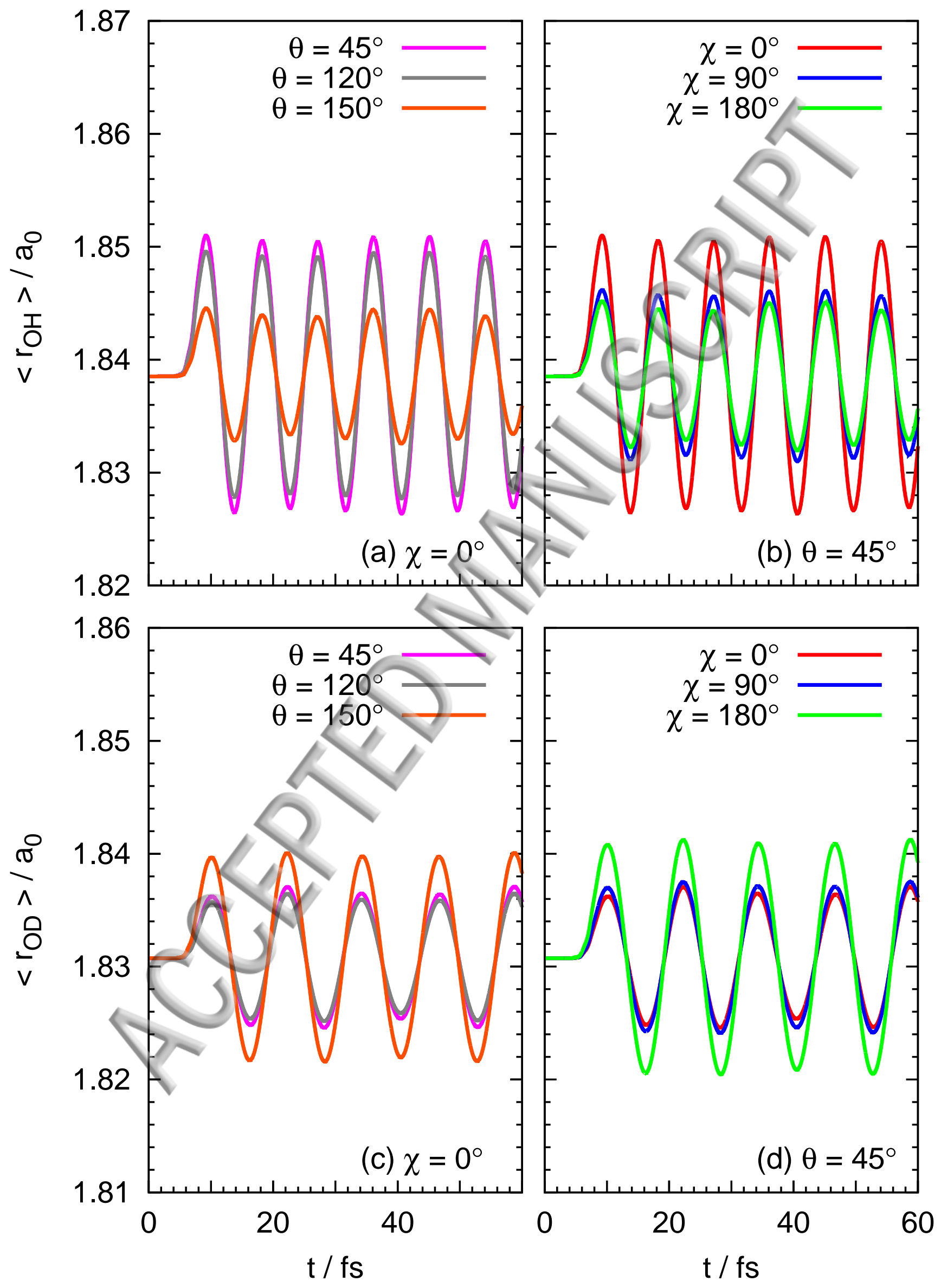


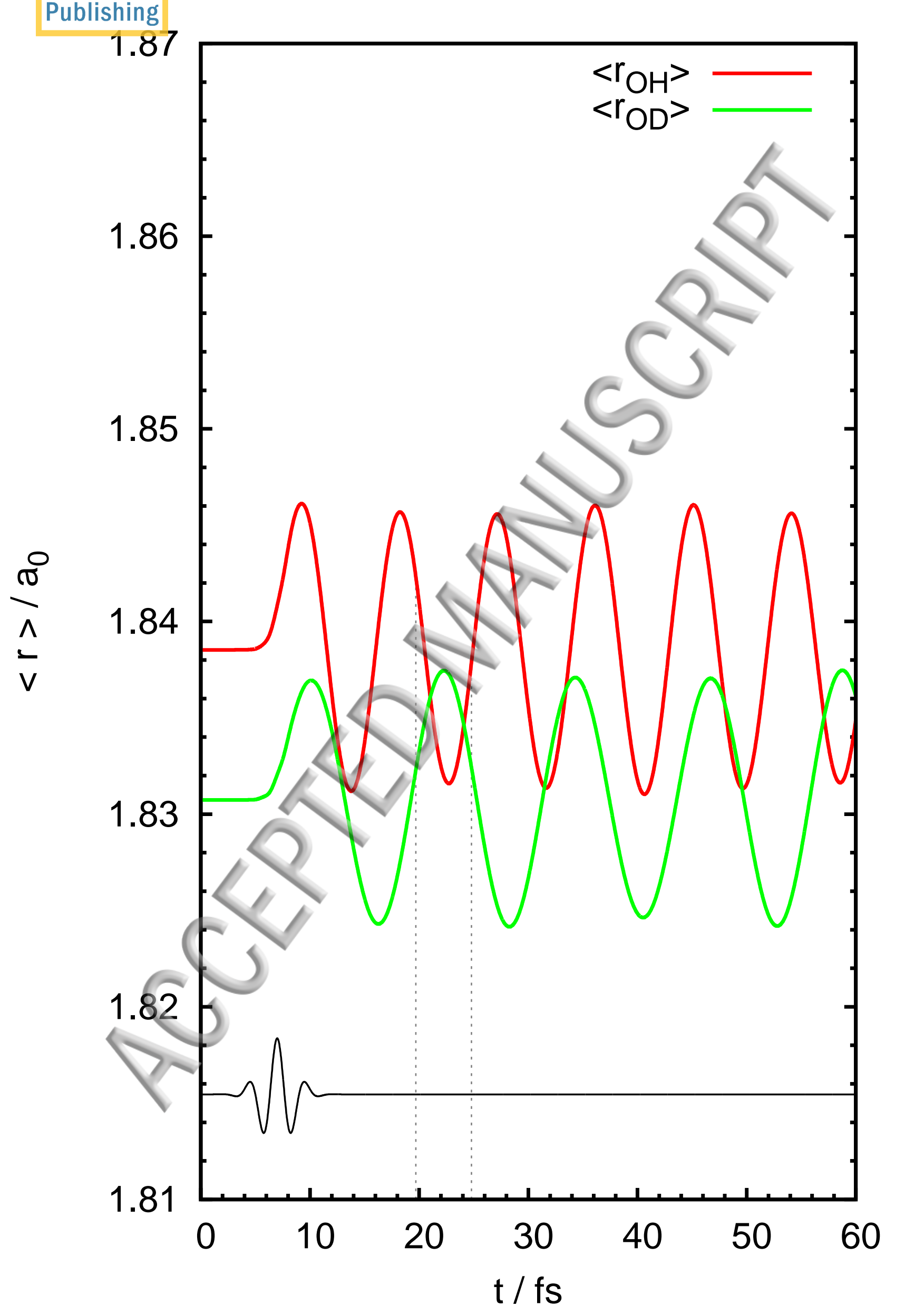




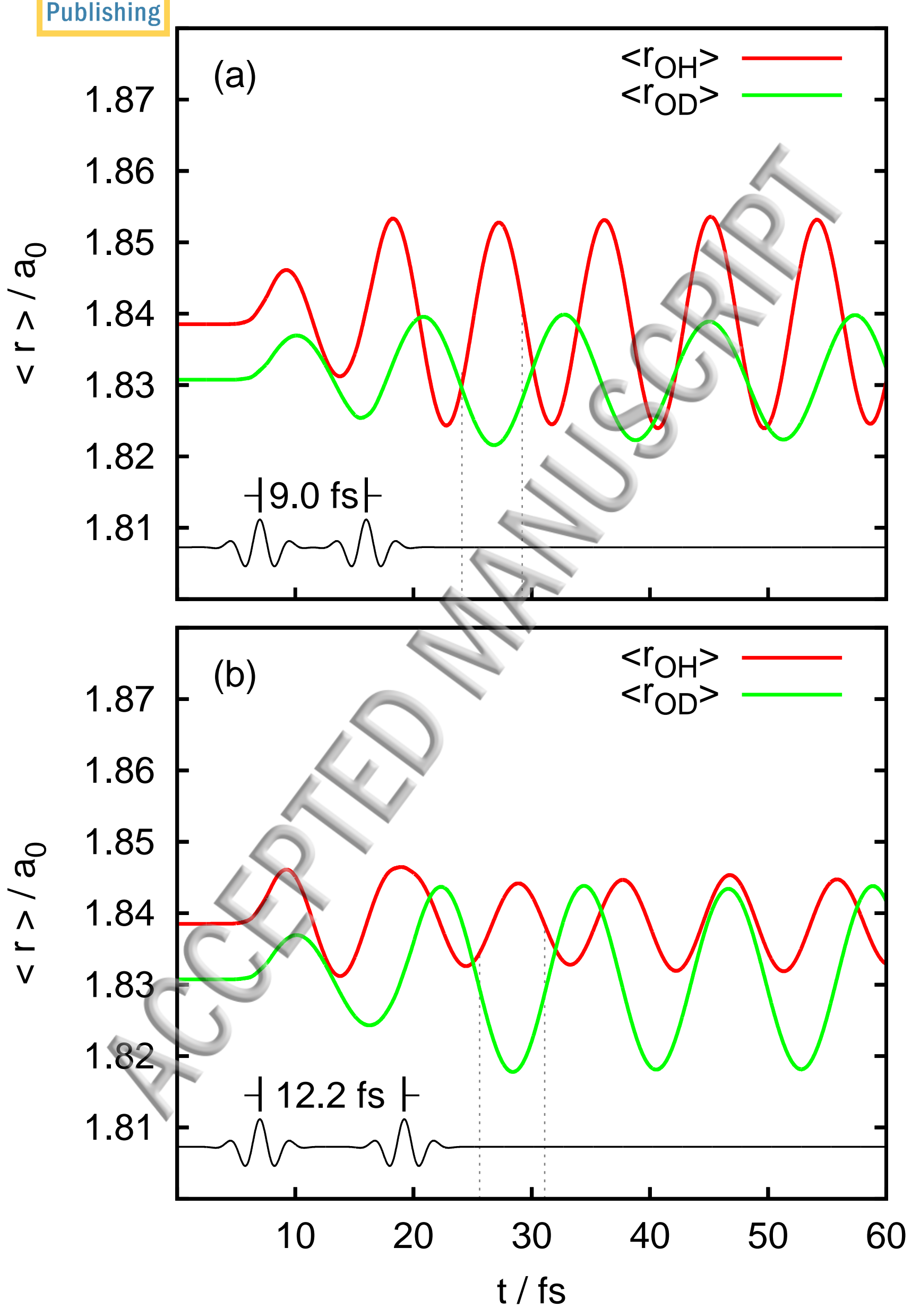



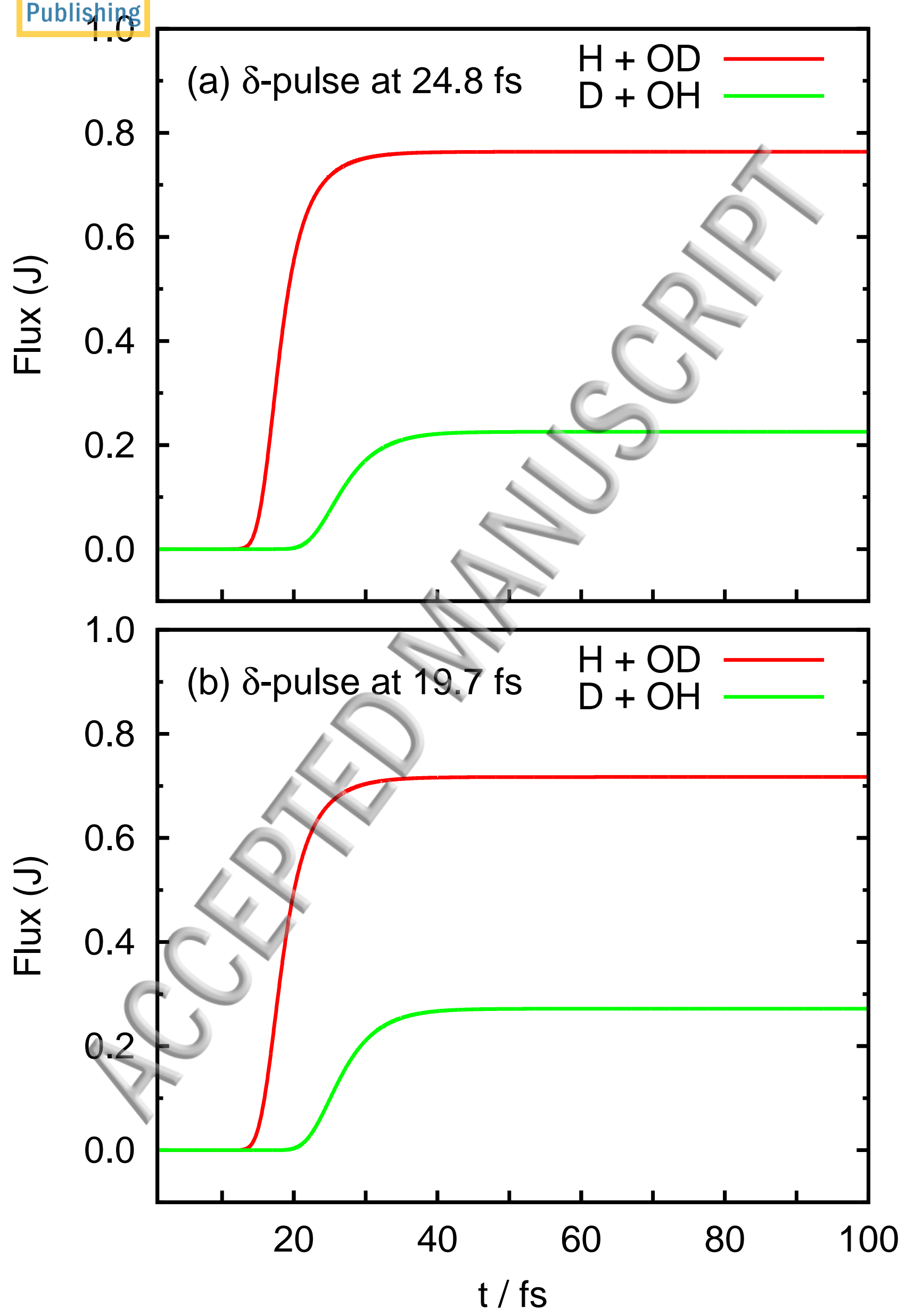
